\title{
Hétéroglossie et écriture dans le roman africançais : le cas d'Ahmadou Kourouma et de Mongo Beti
}

\author{
Rodolphine Sylvie WAMBA et Gérard Marie NOUMSSI \\ Université de Yaoundé I
}

\section{Introduction}

Le concept d'africançais, ainsi qu'il a été précisé dans un précédent essai ${ }^{1}$, caractérise l'écriture du texte africain d'expression française en tant que production littéraire véhiculant la culture d'origine des écrivains avec leurs spécificités esthétiques et normatives. Le lexème apparaît alors comme un paradigme méthodologique d'appoint, dans l'étude esthétique des œuvres littéraires africaines produites en français.

Ce néologisme est composé de deux vocables, «africain » et «français », évoquant deux réalités culturelles en symbiose. Ainsi, du fait de son ambivalence, le terme africançais induit la diglossie. Partant, l'écriture du roman africançais se caractérisera par une hétérogénéité linguistique et/ou stylistique. Chez des auteurs comme Mongo Beti ou Ahmadou Kourouma, ce phénomène est un trait constant de l'écriture ; d'où la problématique de l'écriture romanesque avec un substrat linguistique africain.

S’inspirant des fondements théoriques de Mikhail Bakhtine (1978) pour qui le texte romanesque est par nature plurilingue, la présente analyse tente de cerner les phénomènes d'hétéroglossie langagière dans des textes romanesques d'Ahmadou Kourouma et de Mongo Beti. En effet, dans leurs productions littéraires, ces romanciers problématisent le langage et le donnent à voir. Ce sont ces représentations de la langue qui seront au cœur de nos investigations. On élaborera d'abord le cadre théorique. Par la suite, on procédera à l'analyse des énoncés relatifs à l'hétéroglossie dans notre corpus. Et pour finir, on verra comment ce phénomène participe d'une conception et d'une pratique de l'écriture romanesque chez les romanciers africançais.

\section{Les préalables théoriques}

L'hétéroglossie, dans la perspective bakhtinienne, désigne la présence simultanée de plusieurs langues ou strates langagières dans le texte romanesque. En effet, pour Bakhtine, le langage romanesque se caractériserait d'abord par sa nature hybride; d'où sa dimension polylinguistique. Ainsi envisagée, l'hétéroglossie se manifesterait par «la présence dans un texte d'idiomes étrangers, sous quelque forme que ce soit, aussi bien que de variétés (sociales, régionales ou chronologiques) de la langue principale »(Grutman 37). On a donc affaire à une stylisation du langage romanesque qui peut se réaliser au moins suivant trois modalités.

D’abord la stratification du langage courant, avec ses différentes possibilités textuelles ; il s'agit précisément de

la diversité des langages, et non l'unité d'un langage commun normatif, qui apparait comme la base du style

[...]. Ici le plurilinguisme ne dépasse pas les limites de l'unité linguistique du langage littéraire (selon les

\footnotetext{
1 Ce concept est de Rodolphine Sylvie. Wamba. Elle l'a présenté dans son exposé intitulé «De l'imaginaire linguistique comme moteur de la variation linguistique dans le roman africançais » au colloque des 11,12 et 13 décembre 2008 à Bordeaux sur le thème général «L'imaginaire linguistique dans les textes littéraires, médiatiques et politiques en francophonie ». Les Actes de ce colloque sont sous presses aux Presses Universitaires de Bordeaux en France.
} 
signes verbaux abstraits); il ne devient pas une véritable discordance, il est fixé sur une conception linguistique abstraite, au plan d'un langage unique (c'est-à-dire n'exigeant pas la connaissance de différents dialectes ou langues), mais la compréhension concrète et active [...] du polylinguisme vivant, introduit dans le roman et organisé littérairement, en lui (Bakhtine 129).

Le texte romanesque africançais offre couramment cette forme d'hétérolinguisme, du fait de son ancrage dans des contextes hypoculturels où dominent préalablement une culture, une langue et des groupes sociaux négro-africains avec leurs identités ${ }^{2}$.

Ensuite, une autre forme d'hétérolinguisme consiste en l'introduction des propos de personnages dans le récit littéraire. Ces paroles d'autrui dans un langage étranger peuvent

réfracter les intentions de l'auteur, lui servant, jusqu'à un certain point, de second langage. De plus, les paroles d'un personnage exercent presque toujours une influence (parfois puissante) sur le discours de l'auteur, le parsèment de mots étrangers (discours caché du personnage), le stratifient, et donc y introduisent le polylinguisme (Bakhtine 136).

Cette forme de polylinguisme va jusqu'au psycho-récit qui permet d'obtenir une forme d'hybridation stylistique. Dans ce cas de figure, on assiste à un effacement

de frontières entre le discours de l'auteur et celui d'autrui, grâce à d'autres formes de transmission (discours direct, discours indirect, discours indirect libre), avec leurs multiples combinaisons, et surtout avec divers procédés de leur réplique enchâssée et de leur stratification au moyen du contexte de l'auteur. On parvient [ainsi] au jeu multiple des discours, avec leurs interférences et leurs influences réciproques (Bakhtine 140).

Ces processus sont à l'œuvre dans le roman africançais et permettent de varier la structuration énonciative. Du reste, les propos des personnages rapportés dans le récit romanesque constituent l'espace des manifestations de la coexistence des dialectes propres au contexte sociolinguistique du romancier. Aussi tentera-t-il de rendre compte, dans la langue d'écriture (le français), du répertoire qui est en jeu dans les échanges langagiers de ses personnages, pour autant que ceux-ci manient plusieurs codes linguistiques en fonction des enjeux discursifs; d'où le recours au code switching. On se propose d'analyser ces procédés langagiers au fil de notre réflexion.

Enfin, la forme originale du plurilinguisme dans le roman africançais est l'introduction des genres intercalaires dans la trame narrative. En effet, le roman permet d'intégrer dans son entité toute espèce de genre : proverbe, chant, incantation, etc.

D’après Bakhtine (141), tous ces genres, qui conservent leur élasticité, leur originalité linguistique et stylistique, entrent dans le roman, y introduisent leurs langages propres, stratifiant donc son unité linguistique et approfondissant de façon nouvelle la diversité de ses langages.

Pour ce qui est du corpus, nous avons choisi de nous intéresser aux œuvres de deux romanciers emblématiques de la littérature négro-africaine. En effet, avec Kourouma, on a assisté à une orientation de l'écriture romanesque vers une langue débridée, revendiquant une poétique de l'indigénisation, dont les racines s'étendent jusqu'à l'ethnologie. Par contre, si Mongo Beti incarne un courant puriste, dédaignant le style populaire et prenant ses distances à l'égard de l'africanisation du roman, il n'en demeure pas moins - et de manière paradoxale - que, dans sa dernière trilogie, Alexandre Biyidi a opté pour un style du « roman en liberté », justement incarné par Kourouma. Le résultat est que ces deux auteurs - surtout le Mongo Beti de la dernière

\footnotetext{
2 À en croire Papa Samba Diop (82), l'écriture en contexte hypoculturel essaie, dans ses rapports au français, « de ne pas perdre ses repères linguistiques, géographiques ou historiques »; d'où un usage hybride de la langue d'écriture qui trahit dans tous les cas les références identitaires.
} 
trilogie - présentent à la postérité littéraire une subversion linguistique dont le résultat est une « esthétique du divers ».

Pour ce qui est des exemples, nous mettrons systématiquement en italique les passages pertinents pour nos analyses. Les textes du corpus d'où sont extraites les occurrences seront abrégés comme suit: Les Soleils des indépendances (SI), Branle-bas en noir et blanc (BNB), Trop de soleil tue l'amour (STA), L'Histoire du fou (HF).

\section{Les manifestations littéraires de l'hétéroglossie}

Dans notre corpus, ces manifestations littéraires de l'hétéroglossie bakhtinienne sont particulièrement significatives à trois niveaux: la diversité des langages, matérialisée par une remarquable hybridité discursive, l'enchâssement des propos rapportés et l'organisation des genres intercalaires dans la trame narrative.

\subsection{L'hybridité discursive}

L'hybridité discursive renvoie aux plages textuelles où se manifestent simultanément, dans un même énoncé, la présence de deux instances langagières. Dans le roman africançais, on retrouve couramment une évocation parodique de certaines pratiques langagières de la société africaine qui s'apparentent au commérage. Le récit littéraire se fait alors l'écho de cette «opinion publique ». Il en est ainsi des énoncés suivants dans Les Soleils des Indépendances:

(1) Comme toute cérémonie funéraire rapporte, on comprend que les griots malinkés, les vieux Malinkés, ceux qui ne vendent plus parce que ruinés par les indépendances (et Allah seul peut compter le nombre de vieux marchands ruinés par les indépendances dans la capitale !) «travaillent » tous dans les obsèques et les funérailles (SI 11).

(2) Abdoulaye maraboutait cher. Marabout pour député, ministre, ambassadeur et autres puissants qu'aucune somme ne peut dépasser et qui pourraient se confectionner des pagnes en billets de banque et qui pourtant ne sont pas obligés de prêter à des chômeurs à cause de l'humanisme (SI 66).

À l'analyse, les propos du narrateur des Soleils... se caractérisent par un recours au langage commun de l'opinion publique africaine. En (1), il s'agit de l'idée selon laquelle «les griots malinké » n'ont pas d'autres alternatives que de "travailler dans les obsèques », ruinés qu'ils sont par les indépendances. De même en (2), le narrateur met subtilement sur le compte de l'opinion publique l'idée que certains fonctionnaires post-coloniaux pourraient se tailler «des pagnes à l'aide de billets de banques ».

À l'évidence, il est question pour le narrateur de reproduire «l'attitude verbale normale d'un certain milieu social à l'égard des êtres et des choses; le point de vue et le jugement courant» (Bakhtine 123).

En fait, on a affaire à des constructions qui - d'après les indices grammaticaux (syntaxiques et compositionnels) - appartiennent au seul narrateur, mais qui, en réalité comportent deux énoncés, deux manières de parler; en somme deux langues et deux perspectives sémantiques et/ou sociologiques. Avec Bakhtine (ibid.), on peut y voir "des constructions hybrides ». Il en va ainsi de ces occurrences où les narrateurs d'Ahmadou Kourouma et de Mongo Beti insèrent dans leurs énoncés des propos émanant de la doxa ou des traditions orales : 
(3) En tout, un fils de chef musulman conserve le cœur froid et demeure patient, car à vouloir tout mener au galop, on enterre les vivants, et la rapidité de la langue nous jette dans de mauvais pas d'où l'agilité des pieds ne peut nous retirer (SI 22).

(4) De même que la cellule humaine se positionne de manière à s'accoutumer à l'imprégnation alcoolique pour en devenir un artisan involontaire, de la même façon les populations sédentaires avaient dû s'accoutumer des exactions, des turpitudes des autocrates; elles en avaient pris le pli » (STA 74).

À la lecture de l'énoncé (3), on constate que le narrateur des Soleils... n'approuve pas l'agitation et l'impatience de Fama, prince de son état. Il évoque alors la sagesse populaire aux fins de corroborer sa propre prise de position: agir dans la précipitation peut entraîner à «l'enterrement des vivants», avec toutes les conséquences ce que cela comporte. Enfin, l'on note en (4) une analogie reposant sur l'évocation d'une remarque scientifique dans l'énoncé du narrateur de Trop de soleil tue l'amour: des populations indigènes se sont soumises aux exactions administratives, à l'instar de la cellule humaine devenue complice passive de sa propre destruction par l'alcool.

On ne constate dans ces énoncés, «du point de vue de la composition ou de la syntaxe, aucune frontière formelle. Le partage des voix et des langages se fait dans les limites d'un seul ensemble syntaxique [...] un même discours appartient simultanément à deux langages, deux perspectives qui s'entrecroisent dans cette structure hybride » (Bakhtine 126).

On peut donc voir dans ce type d'énoncé la matérialisation de ce que R. Ludwig et $\mathrm{H}$. Poullet (22) ont appelé "hétéroglossie hybride», signifiant " qu’il y a une véritable interpénétration de registres ou langues [...]. Même si on reconnaît - du point de vue de la réception et de l'analyse - les différentes origines des éléments linguistiques employés, il y a une intention de fusion et de synthèse au niveau de la production ».

Par ailleurs, il est des cas où l'on assiste plutôt à une «motivation pseudo-objective » au sein de l'énoncé. Le résultat est une combinaison de langages dans une même phrase. Dans ce cas, «les conjonctions subordonnées et les conjonctions de coordination (puisque, car, à cause de, malgré, etc.) et les mots d'introduction logiques [...] se dépouillent de l'intention directe de l'auteur, ont un sens étranger, deviennent réfractants, ou même s'objectivent. » (Bakhtine, idem). Le cas est courant dans Les Soleils des indépendances, avec un narrateur qui tourne en dérision le héros du récit.

(5) Les fous, les mendiants et les chômeurs n'ont pas quinze francs; ils ont la pauvreté, le chagrin et la rancœur mais aussi la franchise et l'amitié d'Allah (SI 60).

(6) Donc, pour reconquérir son pouvoir, Fama possédait un sorcier, un griot, de l'argent, des appuis politiques; bref les derniers enthousiasmes de deux vieillards sur leurs derniers pas (SI 144).

En [5], la conjonction mais ne traduit pas un effet de contraste logique entre la pauvreté des marginaux et leur profil moral; au contraire elle permet au narrateur de créer un effet d'ironie au sujet de cette catégorie de personnes prétendument franches et aimables ; alors qu'en réalité, elles sont impitoyables et cruelles au regard de leur comportement dans Les Soleils....Enfin en [6], le narrateur use de la conjonction «donc», pour parodier le prince Fama ruiné par les indépendances et devenu un inadapté social qui compte sur un griot, un sorcier et des appuis politiques fallacieux pour reconquérir son prestige.

Au total, moyennant toutes ces conjonctions détournées de leurs valeurs logiques, on assiste à l'introduction de langages insolites dans le roman, leurs perspectives socio-idéologiques 
trahissant les intentions critiques et satiriques du narrateur. Dans ces conditions, romancier et narrateur construisent du sens puisqu'ils apparaissent « comme vecteurs d'une perspective, d'une vision particulière du monde des événements, d'appréciations et d'intonations particulières » (Bakhtine 132).

Au demeurant, l'écriture romanesque dans notre corpus se caractérise par les interventions constantes des narrateurs. Ainsi, le narrateur des Soleils... prend régulièrement la parole pour expliquer au lecteur la complexité des us et coutumes malinké ou pour le prendre simplement à témoin ; par contre le narrateur de Trop de soleil tue l'amour prend du recul, créant des décalages narratifs qui lui permettent de dégager la moralité des épisodes romanesques. Dans les deux cas, la posture narrative induit l'hétéroglossie, comme le montrent ces exemples :

(7) Le féticheur et sorcier Balla, l'incroyant du village (nous viderons dans la suite le sac de ce vieux fauve, vieux clabaud, vieille byène) rappela à Fama les pratiques d'infidèles (SI 105).

(8) Il alla saluer, se courba, se pencha à la porte de la case où les veuves asseyaient le deuil (pendant quarante jours elles restaient cloitrées) (SI 128).

(9) Pourquoi Eddie avait-il le don de mettre mal à l'aise et même d'exaspérer les gens d'ici - pas tellement l'homme de la rue qui a bien d'autres chats à fouetter, mais les guidams qui, de quelque façon et à tort ou à raison, tiennent le haut du pavé? (STA 73).

L'occurrence [7] comporte une intervention du narrateur des Soleils... dans le récit grâce à une construction incidente dans le but de créer une complicité avec le lecteur au sujet du personnage de Balla ("nous viderons...»). Par contre en [8], il s'agit, pour le narrateur, de proposer au lecteur des explications sur une pratique du deuil propre à la coutume malinké, « asseoir le deuil ». Enfin, en [9], le narrateur de Mongo Beti intervient dans le récit, à l'aide d'une construction incidente, pour fournir des précisions au lecteur sur l'attitude peu vivable du personnage d'Eddie.

Toutes ces interventions du narrateur constituent des enclaves discursives qui créent des strates langagières contrastant avec le récit littéraire originel. De ce fait, ces propos du narrateur apparaissent comme « des discours d'autrui (par rapport au discours direct de l'auteur, qu'il soit réel ou supposé). [...] Et dans ce cas, nous avons sous les yeux un parler indirect non dans un langage, mais au travers d'un langage, au travers d'un milieu linguistique étranger; par conséquent, nous voyons également une réfraction des intentions de l'auteur» (Bakhtine 134). Il convient également de voir comment se réalise la stratification des langages dans les propos rapportés.

\subsection{La gestion des discours rapportés}

Une autre forme de polylinguisme en oeuvre dans le roman africançais réside dans l'introduction et la gestion des propos rapportés. Dans la perspective bakhtinienne, il s'agit de formes de l'hétérophonie (soit la diversité des voix dans l'écriture romanesque).

En effet, la narration de Trop de soleil..., tout comme celle de Branle-bas en noir et blanc, est marquée par la variation diaphrasique et diastratique à cause de la récurrence des dialogues romanesques. Leur récurrence indique une forte oralisation du français. On peut y voir des formes manifestes de l'hétérologie (ou la diversité des registres sociaux et des niveaux de langue). Ainsi, ces textes sont censés restituer fidèlement les parlers populaires du contexte 
urbain camerounais. C'est le cas du dialogue entre deux personnages, Zam et Eddie, dans Trop de soleil tue l'amour :

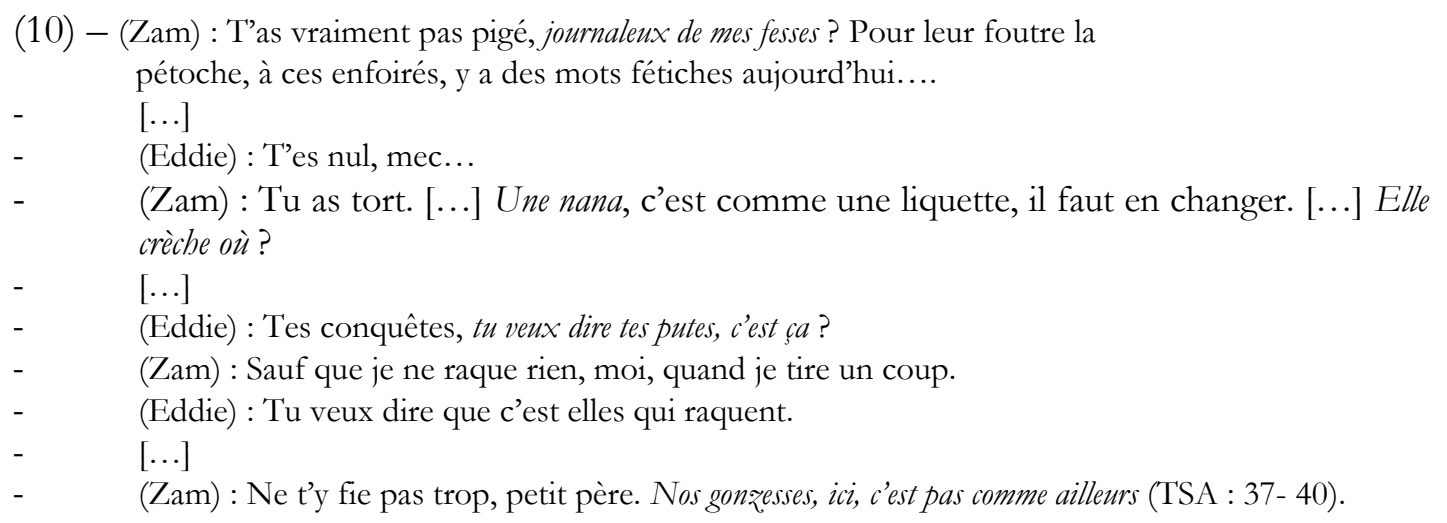

Une observation rapide de ces énoncés révèle le recours à des termes argotiques et à une syntaxe approximative. Soit respectivement: «journaleux de mes fesses », «nana, putes, gonzesses »; « t'es nul, t'as vraiment, c'est ça », etc. Au regard de ce modèle d'écriture, certains critiques ont fait valoir que le français dans les deux derniers romans de Mongo Beti est une langue conforme à la norme du bel usage mais ouverte au français populaire. Et comme le disait Bekri, Trop de soleil....

est avant tout [une] œuvre littéraire où l'écrivain donne à lire des pages merveilleuses de liberté de langage, affranchie de toute pesanteur pédagogique, où les personnages s'adonnent à cœur joie aux paroles les plus succulentes, où les dialogues sont écrits avec une verve salutaire. La langue du roman est vivante, rajeunie, ragaillardie, verdie, charriant les dernières évolutions du langage en France comme en Afrique (119)

Répondant à un critique qui s'interrogeait sur la réception de Trop de soleil... à cause des déviances stylistiques de ce roman, Mongo Beti reconnaitra qu'il a voulu tout simplement prendre le parti de cette langue populaire, «car le français que parlent les Camerounais est une langue très spéciale » (Mongo Beti, cité par Brière 105). Il était déjà convaincu que :

$\mathrm{Si}$ le français doit un jour s'enraciner en Afrique, hypothèse que l'on ne saurait exclure a priori, ce sera comme l'espagnol [sic] en Uruguay ou en Colombie, le Portugais [sic] au brésil, l'anglais [sic] en Amérique, c'est-à-dire en toute liberté, ayant coupé le cordon ombilical qui l'attachait à l'ancienne métropole ou du moins à l'Etat français (Mongo Beti et Tobner 115).

Il faut admettre aussi avec Reboul (187) que « la langue populaire ne témoigne pas d’une incompétence, mais d'une compétence différente. Ainsi, les expressions particulières (fautes ou pas) fonctionnent aussi bien que leurs équivalents de la langue écrite (originale)».

Cette compétence différente est manifeste au niveau interphrastique à travers le code switching. En effet, l'actualisation des propos rapportés donne l'occasion au romancier de recourir au code switching, entendu comme «la juxtaposition, à l'intérieur d'un même échange verbal, de passages où le discours appartient à deux systèmes ou sous-systèmes grammaticaux différents » (Gumperz 57). Animé par un souci d'ordre stylistique, Mongo Beti procède à des alternances codiques interphrastiques.

Dans L'Histoire du fou, c'est la langue latine qui entre en contact avec le français. Le but recherché par Mongo Beti est une certaine exhibition de latinismes connotant la culture très classique de l'auteur. Il en va ainsi dans les exemples suivants :

(11) - Que savez-vous de la sorcellerie ? Rien...Omne ignotum pro magnifico est. [toute chose que l'on ignore est exaltée] Monsieur le Procureur, la sorcellerie est un mythe pitoyable (HF 90). 
(12) - Eh oui, répondit Narcisse. Malheureusement, c'est sans issue de nos jours. Les places sont déjà prises. Tarde venientibus ossa [Nous sommes arrivés tard à la montagne] (HF 50).

Ces énoncés remplissent les critères du code switching: entre autres, charnières syntaxiques nettes, phrases alternées entièrement en latin. On peut voir dans ces occurrences des manifestations de

l'hétéroglossie grotesque, car les composantes de l'hétéroglossie fusionnent d'une part [...] mais d'autre part, les mêmes composantes évoquent leur antithèse linguistico-stylistique. Elles font partie, pour ainsi dire, d'une écriture dialectique dont chaque élément ne peut s'expliquer complètement que par la considération de sa négation » (Ludwig et Poullet 23).

Contrairement à L'Histoire du fou où la superposition des langues met en scène le français et le latin, Trop de soleil...tout comme Branle-bas en noir et blanc offre des plages où «le complexus diglossique » langue africaine (langue ewondo du groupe Beti/Fang au Cameroun) et langue européenne (l'anglais) s’insère harmonieusement dans la chaîne parlée en français. Le résultat de ce processus d'écriture est le recours à la diversité linguistique ou l'hétéroglossie proprement dite, tel que le montrent les exemples ci-après :

(13) - Je ne comprends rien du tout, maugréa la jeune femme dans sa langue maternelle; je ne suis pas une intellectuelle, moi.

- Tu as tort, [...] Tu comprends ça, Bébète ?

- Yё mabissi! [Rien à foutre !] (STA : 13/14).

(14) - Tu te crois dans la cour de ton père ?

- Nna wama! [Par ma mère !] s'écria aussitôt le gros PTC, qui, sous l'effet de la colère, se mettait à parler français à la manière africaine. (STA : 29).

(15)- Oui, d'accord, ékyé, attends un peu, Norbert, reprit le commissaire. (STA : 120).

Si l'auteur prend la peine de donner la traduction des deux emprunts en note de ba » [15], qui est une exclamation assez connue dans l'univers camerounais. Si avec la langue africaine, "dépositaire du génie des Africains », Mongo Beti propose des gloses, il n'en va pas de même avec l'anglais. Visiblement, l'auteur ne s'inquiète pas outre mesure pour la fonction communicative de cette langue généralement véhiculaire en contexte urbain camerounais. Les exemples suivants illustrent ces remarques.

(16) Il n'y a rien à comprendre dans la vie. Il y a le feeling, un point c'est tout. Tu sais ce que c'est ? C'est comme dans le blues, deux ou trois petites notes, un petit verre de gin, et c'est parti. I went down to Saint James Infirmary... (STA 41-42).

(17) Si tu es d'accord, je lui raconte ce qu'il désire entendre, OK ? Et on partage fifty - fifty (STA 100).

(18) Tu es l'homme dont j'ai besoin, en quelque sorte the right man at the right place, tu comprends ça ? (STA 121).

(20) But she wasn't satisfied, she had to run around. Tu entends ça? Jamais contente! Toujours à cavaler à gauche, à droite. Et bien entendu, le cocu, tu sais qui c'est. Non? Tézigue, voyons (STA 159).

Somme toute, que ce soit avec les constructions hybrides, les propos rapportés ou le code switching, on assiste à des cas originaux de métissage linguistique réalisé grâce à des alternances ou des stratifications de structures langagières au sein du texte littéraire. Ce métissage linguistique participe de ce que Ngalasso Mwatha (14) a appelé «diglossie littéraire ». Elle se manifeste par 
des choix linguistiques délibérés et se ramène à des formes de distributions fonctionnelles des langues ou variétés de langues dans l'écriture littéraire. L'énoncé diglossique s'illustrera « fréquemment par l'emploi, à l'intérieur du texte français, de proverbes, dictions, aphorismes en langues africaines [...]. Il apparaît également dans les parties dialoguées lorsque certains personnages parlent une langue africaine, une variété de français [...] ou une langue mixte» (Ngalasso Mwatha (35).

Au demeurant, alors que Mongo Beti aime à créer des effets de théâtralisation dans ses romans à partir des dialogues, Ahmadou Kourouma choisit plutôt de mettre en scène un narrateur-conteur qui établit des relations dialogiques avec le lecteur. Cependant l'originalité narrative de Kourouma réside sans conteste dans l'exploitation du procédé énonciatif du psycho-récit. Il s'agit de la «description par un narrateur omniscient des pensées du personnage mieux que celui-ci ne le ferait lui-même » (Bessonnat 15).

Cette intrusion du narrateur au cœur de la psychologie du personnage s'accompagne d'un changement de ton qui se manifeste par des modalités spécifiques d'énonciation induisant la polyphonie. Il en va ainsi de cet épisode de la mosquée où, à travers la présentation des angoisses de Fama, l'auteur réunit en un tableau le discours impersonnel (la narration) et le discours personnel (l'interrogation et l'exclamation):

(21) [Fama] claqua la langue. Salimata, une femme sans limite dans la bonté du cour, les douceurs des nuits et des caresses, une noire tourterelle [...] Allah! fais, fais donc que Salimata se féconde! ... [...] pourquoi Salimata demeurait-elle toujours stérile. [...]. L'intérieur de Fama battait trouble. Qui pouvait le rassurer sur la pureté musulmane des gestes de Salimata? [...] Blasphème ! gros péché! Fama, ne te voyais-tu pas en train de pécher dans la demeure d'Allah? (SI 27-30).

En somme, l'essentiel du récit des Soleils est présenté à travers la vie intérieure du couple Fama/Salimata, faisant du roman un «texte intérieurement dialogique et complexe, abritant des voix multiples et souvent confondues»(Borgomano 92). Cette multiplicité des voix se réalise aussi par le recours aux formes d'ethno-textes chez l'auteur africançais.

\subsection{L'organisation des genres intercalaires}

Les genres intercalaires se ramènent, dans le récit romanesque, à des textes qui affichent sans ambages leurs spécificités génériques. Ces textes appartiennent à une catégorie littéraire autre, avec leurs modalités esthétiques et thématiques propres. De ce fait, leur insertion dans la prose romanesque n'entraîne nullement l'effacement des traits esthétiques qui les caractérisent. En conséquence, ces genres continueront, même actualisés dans le corps du roman, à résonner de manière originale du fait de leurs langages particuliers. Il en est ainsi, dans l'œuvre de Kourouma, où le contexte d'oralité permet à l'auteur d'insérer des formes originales d'ethnotextes, tels que des proverbes ou des chants dans le récit. Les exemples suivants illustrent ces procédés :

(22) Un enfant : en avoir et le laisser trainer ainsi! L'or ne se ramasse que par celles qui n'ont pas d'oreilles solides pour porter de pesantes boucles (SI 54).

(23) La vérité comme le piment mûr rougit les yeux mais elle ne les crève pas [...] Ton mari, je te le dis d'un intérieur et d'une bouche claire, ne fécondera pas les femmes (SI 76).

(24) Tant que le mur ne se fend pas, les cancrelats ne s'y mettent pas, cancrelats des Indépendances, des partis uniques, de la révolution, vous ne pénétrerez pas, vous ne diviserez pas Togobala (SI 137). 
Généralement le proverbe se caractérise par son caractère imagé et surtout ses contours rythmiques. Pour Julia Sévilla M. (101), « le proverbe est une parémie qui se caractérise par une thématique générique, un sens idiomatique, une structure généralement binaire, des éléments mnémotechniques, une portée universelle »... À cet égard, l'occurrence [22] repose sur une analogie entre «un enfant» (le comparé) et «l'or» (le comparant), le motif commun entre ces deux entités étant naturellement la préciosité. De plus, le trait sentencieux de ce proverbe lui confère un rythme qui est de nature à dynamiser le récit romanesque. Pour ce qui est de l'exemple [23], il s'agit encore d'une analogie : une vérité affligeante à admettre (l'infertilité d'un époux) ; semblable à la douleur du piment qui rougit les yeux. Enfin en [24], on relève, outre la portée rythmique du proverbe, une originalité consistant à dériver cette parémie à partir d'une personnification métaphorique. Le proverbe est énoncé à fin de prémunir les frères de Togobala contre la zizanie (les cancrelats). Dans ces conditions, la moralité, soit l'exhortation à l'union (tant que le mur ne se fend pas), est soulignée avec force.

Il arrive aussi que Kourouma ait recours aux chants dans Les Soleils..., afin de maintenir le rythme de l'oralité. C'est le cas de cet exemple :

(25) Il [Fama] répéta cette mélodie de noce Malinké :

On n'apprécie pas les avantages d'un père, d'un père

Sauf quand on trouve la maison vide du père,

On ne voit pas une mère, une mère

Plus excellente que l'or

Sauf quand on retrouve la case maternelle vide (SI 102).

Outre la disposition en vers libres de cette mélodie, on y distingue un trait stylistique remarquable de l'oralité : les répétitions rythmiques : «d'un père, d'un père » / «d'une mère, d'une mère ».

Au regard de ces formes d'ethno-textes, on peut admettre que les genres intercalaires, bien qu'intégrés dans l'écriture romanesque, conservent leur élasticité, "leur originalité linguistique et stylistique» (Bakhtine 141). Par conséquent, ces ethno-textes importent dans le texte du roman leurs propres langages.

À l'analyse, Kourouma procède à un enchâssement d'énoncés expressifs de la tradition orale dans le roman. L'insertion de ces formules incantatoires permet de conserver le rythme qui est remarquable dans les langues africaines, d'après R. Tro Deho (119).

Du reste, si Kourouma fait constamment appel à l'oralité dans le but de diversifier le langage romanesque, Mongo Beti parvient au même résultat en orientant son œuvre Trop de soleil tue l'amour vers le genre policier. À la lecture de ce texte, on peut alors relever des faits d'expression propres au roman policier. À cet égard, le narrateur de ce texte doit rendre compte du meurtre du père Maurice Mzilikazi.

(26) C'était un prêtre catholique, et pour cette catégorie de personnes, on dit que le suicide est hors de question; mais qu'en savons-nous vraiment? (STA 22)

(27) L'hypothèse du suicide [...] fit long feu, elle jurait trop avec des réalités [...] il apparut que le père Maurice Mzilikeazi avait été assassiné [...] Le meurtre du savant avait été exécuté à la manière d'un crime rituel (STA 23).

Les occurrences précédentes comportent des expressions propres au genre policier: «Hypothèse du suicide»; "(le père M. Mzilikazi) avait été assassiné; le meurtre du savant...crime rituel ». 
De tels faits d'expression corroborent l'idée bakhtinienne selon laquelle les genres intercalaires jouent un rôle constructif dans le roman, du fait de leurs connotations. De fait, le narrateur de Mongo Beti dans Trop de soleil tue l'amour focalise son attention sur les questions d'enquêtes judiciaires, d'où la récurrence des termes de droit ou d'enquêtes policières dans le récit romanesque : «faire le procès de la police et de la magistrature; être chargé d'une enquête; code de procédure criminelle ».

(28) On fit le procès de la police et de la magistrature (STA 67).

(29) Oui, enfin, je suis chargé d'une enquête (STA 122).

(30) P.T.C [était] débouté de sa prétention faute d'une référence explicite au code de procédure criminelle (STA 34).

De tels exemples prouvent à suffisance que le roman de Mongo Beti ouvre une brèche au genre policier. Au surplus, en entrant dans le roman, ce genre y introduit son propre langage, «stratifiant dans son unité linguistique et approfondissant de façon nouvelle la diversité de ses langages » (Bakhtine, idem).

En définitive, l'analyse des phénomènes d'hétéroglossie conduit à une observation : le roman africançais, pris comme un modèle d'écriture, «est un phénomène pluristylistique, plurilingual [et] plurivocal» (Gauvin 172). Cependant, il convient de s'interroger tout de même sur l'attitude des auteurs et l'incidence esthétique du processus.

\section{3- Hétéroglossie et pratique de l'écriture}

Au regard de son profil socio-historique et socio-linguistique, l'écrivain africançais est de fait ancré dans une hétéroglossie qu'il a héritée de sa situation coloniale ; puisque le français, langue d'écriture, n'est pas toujours sa langue maternelle. C'est pourquoi B. Mouralis (165) fait observer que «la communication que l'écrivain africain tente d'établir entre lui et son public naturel n'a pas cessé, loin s'en faut, d'être problématique en raison du médium linguistique ».

On peut alors s'attarder sur l'attitude épilinguistique des auteurs, pour ce qui est de leurs processus d'écriture dont le but ultime est la création d'une esthétique de l'hybridité. En effet, Kourouma (137) estimait que l'objectif «recherché par le créateur dans la tradition négroafricaine est de favoriser la participation par l'émotion. Il y parvient en usant du rythme, de l'image et du symbole comme procédés littéraires ». Dans cette perspective, le romancier ivoirien constatait qu'à la langue de l'ex-colonisateur, il manque «le lexique, la grammaticalisation, les nuances et même les procédés littéraires pour lesquels l'œuvre avait été préparée » (Kourouma, idem).

C'est ainsi que l'auteur ivoirien justifiera a posteriori la subversion du français dans son écriture romanesque. Cette subversion dans Les Soleils... passe par le recours systématique à des tournures considérées comme fautives, proches de la désinvolture stylistique et de nature à subvertir la langue française : «nuitez »; « assois tes fesses »; « bâtard de bâtardise ».

(31) Fama se récriait : «Bâtard de bâtardise! Gnamokodé!» (SI 11)

(32) Les palabreurs comprirent que le sommeil avait conquis ses paupières. « Nuitez en paix... » (SI 96). 
(33) C'est à cet instant que fusa de l'assemblée l'injonction : - Assois tes fesses et ferme la bouche! (SI 15).

Au surplus, l'auteur de Monnè... aura constamment recours aux us et coutumes malinké, comme s'il tenait à articuler l'œuvre littéraire à son contexte ethnologique. Il en va ainsi de cette formule rituelle qui situe le lecteur au premier et dernier chapitre des Soleils... sur la pratique des funérailles malinké.

(34) Des jours suivirent le jour des obsèques jusqu'au septième jour et les funérailles du septième jour se déroulèrent devant l'ombre, puis [...] arriva le quarantième jour, et les funérailles du quarantième jour ont été fêtées au pied de l'ombre accroupie (SI : 10).

À partir de l'actualisation d'un tel énoncé propre aux traditions orales malinké dans son roman, Kourouma fait œuvre d'ethnologie religieuse et affiche une attitude de passeur culturel. Une telle démarche se réalise par le recours à une forme rituelle de langage. Le processus relève de ce que Zabus (1991) a appelé "relexification», entendu comme une reproduction délibérée des expressions de la langue maternelle dans la syntaxe du français : relexification « best describes the process at work when the African Language is simulated in the Europhone text. [...] The emphasis here on the lexis in the original sense of speech, word or phrase and on lexicon in reference to the vocabulary and morphemes of a language » (Zabus 101-102).

C'est ce processus d'écriture qui permet à Kourouma d'user systématiquement de palimpsestes verbo-culturels dans Les Soleils... tel qu'on le remarque dans ces énoncés :

(35) Et Mariam était une femme ayant un bon ventre, un ventre capable de porter douze maternités (SI 130).

(36) Bamba ! S’égosillait-il ; refroidissez le cour! (SI 16).

(37) Et arrivait l'heure de la troisième prière, troisième prière de ce jour que Fama devait courber sur la tombe (SI 114).

Ces termes (en italique) montrent bien qu'on est en présence d'une interlangue, ou troisième registre, trahissant la subversion linguistique du romancier; d'où un processus d'indigénisation de la langue française, qui permet à Kourouma de « créer sa propre langue dans la langue, d'en déplacer les frontières et de les pousser au-delà des limites convenues » (Gauvin $9)$.

Contrairement à Kourouma, l'attitude de Mongo Beti repose davantage sur une volonté « de substituer à une image ethnologique de l'Afrique une vision sociologique qui fasse apparaittre les tensions et les conflits dont celle-ci est le théâtre [...], dans les Etats issus de la colonisation » (Mouralis 158). D'une certaine manière, un tel engagement passe par un travail sur le langage romanesque. L'auteur fait d'ailleurs observer: «je constate chaque jour que, au lieu de me rapprocher de mon peuple [...], ce qui est la fonction naturelle de la langue d'un écrivain, la langue française, au contraire, m'en éloigne... » (Mongo Beti 237). Peut-on penser que le style et la pratique de l'écriture adoptée dans Trop de Soleil tue l'amour ou Branle Bas en Noir et Blanc, avec une survalorisation des parlures populaires dans le texte du roman, aient été motivés par cet infléchissement stylistique? Tout porte à le croire, au regard de la prise de conscience linguistique ayant précédé la parution de ces deux derniers romans d'Alexandre Biyidi.

De fait, les deux derniers romans de Mongo Beti se caractérisent par une grande diversité des registres langagiers, avec des expressions du français camerounais, et ce, malgré les interventions du narrateur dans un style généralement soutenu. Ces occurrences du français 
camerounais contrastant avec le style soutenu du narrateur induisent l'hétéroglossie comme le montrent ces exemples :

(38) D'abord il ne prend jamais de risque ni en parole ni quand vous faites des marches [manifestations de rue] (STA 51).

(39) Cinq mille! avait claironné le marchand ambulant, appelé ici samveteur. (STA 149).

(40) Qui t’a même appelé ? [...] Tu viens là, tu trouves que les gens parlent leur affaire, et tu mets ta bouche (STA 149).

Du reste, à la lecture des dernières œuvres de Mongo Beti, on constate que de telles parlures actualisées dans l'écriture romanesque induisent une véritable subversion de la langue française ; car même si la narration frappe par son style soigné, elle «alterne avec de nombreux dialogues qui généralement reposent sur l'emploi d'un tout autre registre de style : textes censés reproduire un parler populaire [...] ou des propos censés être tenus dans une langue africaine » (Mouralis 157).

Somme toute, que ce soit chez Mongo Beti ou Kourouma, à travers la subversion de la langue d'écriture, on assiste à de véritables mises en scène de la langue telles qu'elles apparaissent dans l'espace des textes littéraires : «mises en scène de type réflexif fournies par les préfaces [...] ou manifestes divers; mises en scène textualisées qu'offrent les œuvres elles-mêmes et les dispositifs langagiers sur lesquels elles s'appuient » (Gauvin 8).

On est donc conduit à parler d'une surconscience linguistique chez les romanciers africançais ;

conscience de la langue comme lieu de réflexion privilégiée, comme territoire imaginaire à la fois ouvert et contraint [...] [Ainsi], le choix de telle ou telle langue d'écriture est révélateur d'un procès littéraire plus important que les procès en jeu. Plus que de simples modes d'intégration de l'oralité dans l'écrit, ou que la représentation plus ou moins mimétique des langages sociaux, on dévoile ainsi le statut d'une littérature et son intégration/définition des codes (Gauvin 256).

\section{Conclusion}

Comme concept d'analyse en stylistique ou en littérature, l'hétéroglossie se trouve au cœur de plusieurs possibles scripturaux : entre autres l'hybridité discursive, le discours rapporté et les genres oraux. Non seulement ce concept prend en compte le phénomène de plurilinguisme, mais également il se décline dans l'approche de Bakhtine en hétérologie et hétérophonie. Dans tous les cas, il s'agit de désigner des pratiques langagières dans des contextes d'écriture où se manifeste une distribution fonctionnelle des langues ou des variétés de langues en fonction des enjeux esthétiques. C'est ce que nous avons illustré avec l'analyse de notre corpus. Ce phénomène est d'autant plus saillant chez l'écrivain africançais, qui se situe lui-même au carrefour de plusieurs langues et qui est par là même directement impliqué dans l'hétéroglossie. Partant, il doit construire et négocier son propre langage dans une dialectique faite d'appropriation/créativité des ressources langagières. Il en résulte nécessairement un discours hybride, voire métissé, dont l'espace romanesque est le champ de prédilection. Dès lors que ce processus n'est pas subi, mais assumé par les auteurs eux-mêmes, on peut y voir le reflet d'une surconscience linguistique. 


\section{Bibliographie}

\section{Corpus}

Beti, Mongo. Branle-bas en noir et blanc. Paris : Julliard, 2000.

--.. Trop de soleil tue l'amour. Paris : Julliard, 1999.

---. L'Histoire du fou. Paris : Julliard, 1994.

Kourouma, Ahmadou. Les Soleils des indépendances. Paris : Le Seuil, 1970.

\section{Ouvrages théoriques}

Bakhtine, Michail. Esthétique et théorie du roman. Paris : Gallimard, 1978.

Bessonnat, Daniel. "Paroles de personnages : problèmes, activités d'apprentissage." Pratiques 65 (1990) : 7-35.

Borgomano, Madeleine. Ahmadou Kourouma. Le «Guerrier» griot. Paris : L'Harmattan, 1998.

Brière, Eloïse. "Lire, enseigner et rencontrer Mongo Beti." Remember Mongo Beti. Bayreuth : African Studies, 2003. 101-107.

Gauvin, Lise. La Fabrique de la langue. Paris : Le Seuil, 2004.

Grutman, Rainier. Des langues qui résonnent. L'bétérolinguisme au XIX siècle québecois, Montréal: Fides-CETUQ, 1997.

Gumperz, John. Sociolinguistique interactionnelle. Une approche interprétative. Paris : L'Harmattan, 1989.

Kourouma, Ahmadou., "Le Processus d'Africanisation des langues européennes." Nowvelles du Sud 26 (1997) : 135-139.

Ludwig, Ralph et Poullet, Hector. "Langues en contact et hétéroglossie littéraire : l'écriture de la créolité." CCLWeb: http://www.montraykreyol.org/IMG/pdf/Ludwigpoullet12.pdf,

(2007) : 1-27 .Web. 08 juillet 2010.

Mongo, Beti. "L’Ecrivain francophone, le public, la société. " Nouvelles du Sud 26 (1997) : 237242.

Mongo, Beti et Tobner, Odile. Dictionnaire de la négritude. Paris : L'Harmattan, 1989.

Mouralis, Bernard. Littérature et développement. Essai sur le statut, la fonction et la représentation de la littérature négro-africaine d'expression française. Lille : atelier de reproduction des thèses, 1981.

Mouralis, Bernard. "Le classicisme de Mongo Beti." Littératures Francophones : Langues et styles. Ed. Centre d'études francophones. Paris: L'Harmattan, 2001. 155-166.

Ngalasso Mwatha, Musanji "Langues, Littératures et cultures africaines." Recherches et travaux 27 (1984) : 21-39.

Noumssi, Gérard Marie et Wamba, Rodolphine Sylvie. "Créativité esthétique et enrichissement du français dans la prose romanesque d'Ahmadou Kourouma." Présence Francophone 59 (2002) : 28-51.

Noumssi, Gérard Marie. La Créativité langagière dans la prose romanesque d'Ahmadou Kourouma. Paris : L'Harmattan, 2009.

Reboul, Olivier. Qu'est-ce qu'apprendre? Paris : PUF, 1991.

Samba Diop, Papa. Archéologie littéraire du roman sénégalais. Iko: Verlag für Interkulturelle kommunikation, 1993.

Sevilla Muñoz, Julia. "Les Proverbes et les phrases proverbiales français et leurs équivalences en espagnol." Langages 139 (2000) : 98-109.

Tro Deho, Roger. Création romanesque négro-africaine et ressources de la littérature orale. Paris : L'Harmattan, 2005.

Van den Avenne, Cécile. "Passer d'un monde à l'autre, d'une langue à l'autre - Lecture de deux incipits d'Ahmadou Kourouma." Rpt. Dans Plurilinguisme et apprentissage: Mélanges Daniel Coste. Ed. Mochet M.A. et al. Paris : ENS-Editions, 2005. 1-7. 
Wamba, Rodolphine Sylvie. "Trop de soleil tue l'amour: une expression de l'écriture du mal-être de Mongo Beti." Présence Francophone 63 (2004) : 168-188.

Zabus, Chantal. The African palimpsest: Indigenization of Language in the West African Europhone Novel. Amsterdam - Atlanta: Rodopi, 1991. 